\title{
Approximate analytic solution of heat conduction in hollow semi-spheres flying at hypersonic speed ${ }^{2}$
}

\author{
Guilai Han *, Zonglin Jiang \\ Stake Key Laboratory of High Temperature Gas Dynamics (LHD), Institute of Mechanics, Chinese Academy of Sciences (CAS), Beijing, 100190, China
}

\section{A R T I C L E I N F O}

Available online 15 February 2013

\section{Keywords:}

Heat conduction

Theoretical investigation

Approximate solution

Hypersonic

\begin{abstract}
A B S T R A C T
Heat transfer in blunt noses of hypersonic vehicles with coolant inside can be approximately considered as heat conduction in hollow semi-sphere with aerodynamic heating on the outer boundary and enhanced cooling on the inner boundary. Theoretical investigations of temperature field in hollow semi-spheres were carried out by solving the two-dimensional axsymmetric conduction equation, which could be transformed into Legendre equation when the separation of variables is applied. However, for such a semi-sphere flying at hypersonic speed, the distribution of heat transfer rates as an outer boundary condition is so complex that the integration in the Legendre solution is nearly impossible to be completed. In this paper, a 4th order Legendre polynomial, derived by the method of undetermined coefficients, was adopted to approach the local similarity solution of hypersonic aerodynamic heating and simplify the integration process, by which an approximate solution could be set up for the temperature field. The approximate solution is also validated by comparing the analytical results with data from numerical simulations, in which the conduction equation is solved with the improved Richardson scheme. Both analytical and numerical results are compared to each other and match quite well.
\end{abstract}

(c) 2013 Elsevier Ltd. All rights reserved.

\section{Introduction}

For hypersonic vehicles, serious aerodynamic heating is a great barrier. Both heat transfer rates at local positions and their integration over surface and flight time are considered to be governing factors for the design of thermal protection systems (TPS) [1]. Usually, it is difficult for researchers to get a clear figure on such a physical phenomenon due to the complexity of aerodynamic heating by hypersonic flow and conduction inside the structure. During the last two decades, numerical simulations have led to some better understanding of physical mechanisms and applications in engineering, such as the coupling of aerodynamic heating and structural conduction, enhanced convective cooling et al. However, as effective ways for scientific researches, it is usually not easy for numerical simulations or experiments to show the regularity between parameters, such as temperature field, flow field, dimension, and heat transfer rates et al. In the latest decade, plenty of investigations were dedicated to regenerative cooling of hypersonic vehicles, and the most typical examples are thermal protection systems for propulsion systems, such as scramjets [2]. The aerodynamic heating or heat transfer in engines due to combustion will result in high heat flux into structure. If the structural temperature went too high, the performance of material, structure, and surface coating, such as oxidation resistance, strength, cooling efficiency et al.,

\footnotetext{
is Communicated by W.J. Minkowycz.

* Corresponding author. Tel: +86 10 82545730; fax:+86 1082544145. E-mail address: hanguilai@imech.ac.cn (G. Han).
}

will get significant discounts, which may result in serious damage to the vehicles. Usually, for regenerative cooling, fuel is used as coolant to absorb the heat and lower the temperature of structure in hypersonic air-breathing vehicles. During the cooling process, fuel gets preheated and may lead to higher combustion efficiency due to phase changing and fuel cracking happening during the fuel heating.

In this paper, the model was set as a hollow semi-sphere flying at hypersonic speed with convective cooling inside, as shown in Fig. 1(a). Thereby, the heat conduction in the semi-sphere is the main subject in the following chapters. To simplify the investigations, the high temperature environment generated by the hypersonic coming flow is replaced by an aerodynamic heating distribution on the outer boundary, which is an analytical form governed by the local similarity solution [3]. By assuming the inner convective cooling inside with efficiency high enough, the temperature of the inner wall is much lower than the critical value, which may result in discount of material and structural strength, oxidation resistance, or ablation of surface coating. Therefore, the governing equation and ideal model can be shown as Eq. (1), which is also depicted in Fig. 1(b)

$\frac{1}{r^{2}} \frac{\partial}{\partial r}\left(r^{2} \frac{\partial T}{\partial r}\right)+\frac{1}{r^{2} \sin \theta} \frac{\partial}{\partial \theta}\left(\sin \theta \frac{\partial T}{\partial \theta}\right)=0$

According to the hypothesis made before, the boundary conditions for Eq. (1) can be shown as follows

$\left.\frac{\partial T}{\partial \theta}\right|_{\theta=0}=\left.\frac{\partial T}{\partial \theta}\right|_{\theta=\pi / 2}=0$ 


\section{Nomenclature}

$A_{\mathrm{n}}, B_{\mathrm{n}} \quad$ coefficients of series or polynomial

$f(\theta) \quad$ normalized heat rate on outer boundary

$g(\theta) \quad$ approximate normalized heat rate on outer boundary

$M, M_{\infty} \quad$ Mach number of hypersonic incoming flow

$P_{\mathrm{n}} \quad$ first class Legendre function

$Q_{n} \quad$ second class Legendre function

$r, \theta \quad$ axis of spherical coordinate system

$r_{1}, r_{2} \quad$ inner and outer radius of hollow semi-sphere

$T \quad$ temperature field of hollow semi-sphere

$T_{\mathrm{n}} \quad$ series form of $T$

$T_{\mathrm{w}} \quad$ temperature distribution on inner boundary

$x, y \quad$ axis of Cartesian coordinate system

n natural number

i No. of the grid point for the finite difference method

\section{Greek letters}

$\Delta t \quad$ time step

$\Gamma \quad \Gamma$ function

$\alpha \quad$ ratio between $r_{2}$ and $r_{1}$

$\varepsilon \quad$ circumferential motion factor

$\xi, \eta \quad$ axis of computational space coordinate system

$\gamma \quad$ Euler number

$\gamma_{\infty} \quad$ specific heat ratio of incoming flow $q_{\mathrm{w}} \quad$ aerodynamic heat rate distribution on outer boundary

$\Delta x \quad$ space step

$\beta \quad$ coefficient of parabolic equation

integration process in the original analytical solution possible. Further, the governing equations with boundary conditions as depicted in Eqs. (2) to (4) was numerically solved by a finite difference method with the improved Richardson scheme. Both analytical and numerical solutions match with each other, so the approximate analytical solution can be considered as demonstrated and validated. Details and further discussions about the applications of such a solution will be represented in the following chapters.

\section{Theoretical solution}

The solution of Eqs. (1) to (4) is not very complex, which might be found in some textbooks or reference books. For the convenience of understanding and checking such a paper, a few key procedure of the derivation process will be included in the following. With separation of variables, the temperature field can be defined as $T(r, \theta)=R(r) \Phi(\theta)$, and Eq. (1) could be transformed in to a typical eigenvalue problem with two equations shown as follows

$\frac{1}{\sin \theta} \frac{d}{d \theta}\left(\sin \theta R \frac{d \Phi}{d \theta}\right)+\lambda \Phi=0$

$\frac{d}{d r}\left(r^{2} \frac{d R}{d r}\right)-\lambda R=0$

where $\lambda=n(n+1)$, and $n=0,1,2 \ldots$

Eqs. (5-a) and (5-b) can be solved individually with linear superposition for the final solution. If the transformations as $\tau=\cos \theta$ and $\varphi(\tau)=\Phi(\theta)$ were applied and substituted into Eq. (5-a), and the following equation could be obtained

$\frac{d}{d \tau}\left[\left(1-\tau^{2}\right) \frac{d \varphi}{d \tau}\right]+n(n+1) \varphi=0$

$\left.T\right|_{r=r_{1}}=T_{\mathrm{w}}(\theta)$

$\left.\frac{\partial T}{\partial r}\right|_{r=r_{2}}=q_{\mathrm{w}}(\theta)$

Since the solution of Eq. (1) will be in form of the Legendre function [4], and the integration process is very difficult to operate, this paper will find out an approximate analytical solution by using a 4th order Legendre polynomial to approach the local similarity solution for aerodynamic heating in hypersonic flight, which makes the
This equation is classic Legendre equation, and the generalized solution in the neighborhood of $\tau=1(\theta=0)$ can be stated as

$\varphi(\tau)=\mathrm{C}_{1} P_{\mathrm{n}}(\tau)+\mathrm{C}_{2} Q_{\mathrm{n}}(\tau)$

where $P_{\mathrm{n}}(\tau)$ and $Q_{\mathrm{n}}(\tau)$ represent the first and second class of Legendre polynomials, respectively. The detailed expressions are listed as following

$P_{\mathrm{n}}(\tau)=\sum_{k=0}^{n} \frac{1}{(k !)^{2}} \frac{\Gamma(n+k+1)}{\Gamma(n-k+1)}\left(\frac{\tau-1}{2}\right)^{k}$

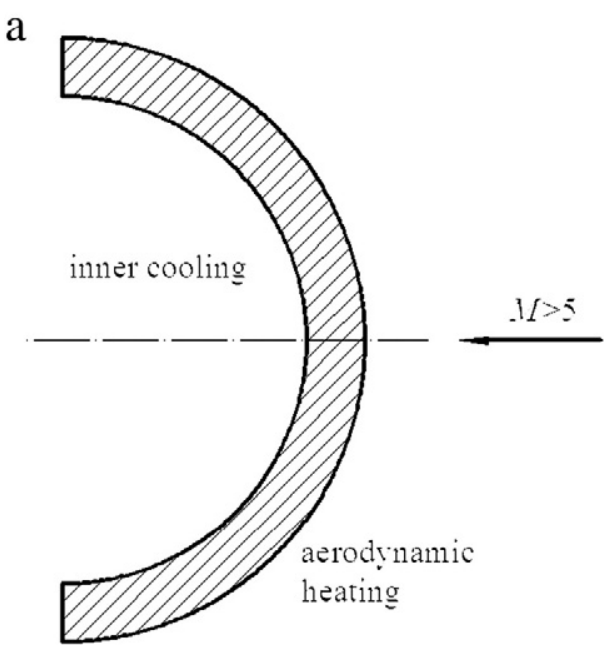

b

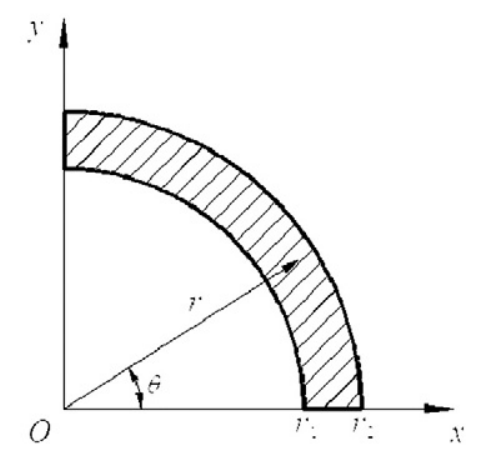

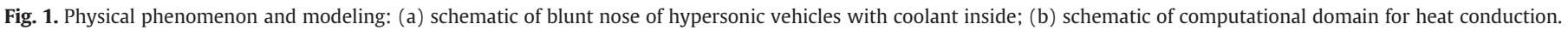




$$
\begin{aligned}
Q_{\mathrm{n}}(\tau)= & \frac{1}{2} P_{\mathrm{n}}(\tau)\left[\ln \frac{\tau+1}{\tau-1}-2 \gamma-2 \psi(n+1)\right] \\
& +\sum_{k=0}^{n} \frac{1}{(k !)^{2}} \frac{\Gamma(n+k+1)}{\Gamma(n-k+1)}\left(1+\frac{1}{2}+\ldots+\frac{1}{n}\right)\left(\frac{\tau-1}{2}\right)^{k}
\end{aligned}
$$

In Eqs. (8-a) and (8-b), $\gamma$ denotes the Euler number while $\psi(\mathrm{n}=1)$ stands for the log scaled differential quotient of the $\Gamma$ function. Since $\varphi(\tau)$ has a limitation at the position of $\tau=0(\theta=\pi / 2)$, the coefficient for $Q_{\mathrm{n}}(\tau)$ can be derived as $C_{2}=0$. Therefore, the solution of $\Phi(\theta)$ can be shown in form of series, like

$\Phi_{\mathrm{n}}(\theta)=P_{\mathrm{n}}(\cos \theta)$ by

The differential calculus of $\Phi_{n}(\theta)$ in Eq. (9) over $\theta$ can be obtained

$\frac{d \Phi_{n}}{d \theta}=\frac{d P_{n}(\cos \theta)}{d \theta}=-P_{n}^{\prime}(\cos \theta) \sin \theta$

Hence, the boundary conditions stated in Eq. (2) can be written as

$\left.\frac{d \Phi}{d \theta}\right|_{\theta=0}=0$

and

$\left.\frac{d \Phi}{d \theta}\right|_{\theta=\pi / 2}=0$

For Eq. (11-a), $\sin \theta$ becomes 0 when $\theta=0$, which means the boundary condition at position of $\theta=0$ can be satisfied permanently. In contrast, the Legendre function cannot be analytic at the location of $\tau=0(\theta=\pi / 2)$ due to the shortage itself. Hence, the constraint of the boundary condition given in Eq. (11-b) is neglected here momentarily for convenience of further discussions.

With transformation applied, the generalized solution of Eq. (5-b) can be got

$R_{\mathrm{n}}(r)=A_{\mathrm{n}} r^{\mathrm{n}}+B_{\mathrm{n}} r^{-(\mathrm{n}+1)}$

Combining Eqs. (9) and (12), it will get the solution of the temperature field in the form of series can be retrieved as shown in Eq. (13).

$T_{\mathrm{n}}(r, \theta)=R_{\mathrm{n}}(r) \Phi(\theta)=\left[A_{\mathrm{n}} r^{n}+B_{\mathrm{n}} r^{-(\mathrm{n}+1)}\right] P_{\mathrm{n}}(\cos \theta)$

where $n$ equals $0,1,2 \ldots$

Since the conduction Eq. (1) along with the boundary conditions (Eqs. (2) to (4)) is linear, the superposition method can be applied to construct homogeneous boundary conditions for the determination of the coefficients in Eq. (13). Then the solutions under homogeneous boundary conditions can be superposed with each other to obtain a solution of the entire temperature field. In doing so, the boundary conditions can be constructed as follows

$\left.T\right|_{r=r_{1}}=T_{\mathrm{w}}(\theta),\left.\frac{\partial T}{\partial r}\right|_{r=r_{2}}=0$

and

$\left.T\right|_{r=r_{1}}=0,\left.\frac{\partial T}{\partial r}\right|_{r=r_{2}}=q_{\mathrm{w}}(\theta)$
Substituting the generalized solution of Eq. (13) into Eq. (14-a), the following coefficients can be obtained.

$\left\{\begin{array}{l}A_{\mathrm{n}}=\frac{1}{r_{1}^{\mathrm{n}}\left[1+\frac{\mathrm{n}}{\mathrm{n}+1}\left(\frac{r_{2}}{r_{1}}\right)^{\mathrm{n}}\right]} \frac{2 n+1}{2} \int_{0}^{\pi} T_{\mathrm{w}}(\theta) P_{\mathrm{n}}(\cos \theta) \sin \theta d \theta \\ B_{\mathrm{n}}=\frac{n}{n+1} r_{2}^{2 n+1} A_{\mathrm{n}}\end{array}\right.$

Similarly, substituting Eq. (13) into Eq. (14-b) yields to

$\left\{\begin{array}{l}A_{\mathrm{n}}=\frac{1}{r_{2}^{n-1}\left[n+(n+1)\left(\frac{r_{1}}{r_{2}}\right)^{2 n+1}\right]} \frac{2 n+1}{2} \int_{0}^{\pi} q_{\mathrm{w}}(\theta) P_{\mathrm{n}}(\cos \theta) \sin \theta d \theta \\ B_{\mathrm{n}}=-r_{1}^{2 n+1} A_{\mathrm{n}}\end{array}\right.$

With the coefficients determined, the series solution of the temperature field can be stated as

$$
\begin{aligned}
T(r, \theta)= & \sum_{n=0}^{\infty} \frac{2 n+1}{2}\left[\left(\frac{r}{r_{2}}\right)^{n}+\frac{n}{n+1}\left(\frac{r_{2}}{r}\right)^{n+1}\right] P_{\mathrm{n}}(\cos \theta) \frac{(n+1) \alpha^{n}}{n+1+n \alpha^{2 n+1}} \int_{0}^{\pi} T_{\mathrm{w}}(\varphi) P_{\mathrm{n}}(\cos \varphi) \sin \varphi d \varphi \\
& +\sum_{n=0}^{\infty} \frac{2 n+1}{2}\left[\left(\frac{r}{r_{1}}\right)^{n}-\left(\frac{r_{1}}{r}\right)^{n+1}\right] P_{\mathrm{n}}(\cos \theta) \frac{r_{2} \alpha^{-n}}{n+(n+1) \alpha^{-(2 n+1)}} \int_{0}^{\pi} q_{E}(\varphi) P_{\mathrm{n}}(\cos \varphi) \sin \varphi d \varphi
\end{aligned}
$$

where $\alpha=r_{2} / r_{1}$ denotes the ratio between outer and inner radii. However, as mentioned before, the Legendre function $P_{\mathrm{n}}(\cos \theta)$ cannot be analytic when $\theta$ equals to $\pi / 2$, and the boundary constraint was neglected momentarily to enable solution. Therefore, the solution of temperature field in Eq. (17) might differ from the physical solution or reality, especially nearby the location of $\theta=\pi / 2$. Hence, the comparison between the analytical and numerical solutions will be handled in the next chapter.

\section{Numerical simulation}

For convenience, the two-dimensional axsymmetric unsteady heat conduction equation is applied to simulate the process. The non-dimensionalized form in the Cartesian coordinate system can be shown as

$\frac{\partial T}{\partial t}=\frac{\partial^{2} T}{\partial x^{2}}+\frac{\partial^{2} T}{\partial y^{2}}+\frac{1}{y} \frac{\partial T}{\partial y}$

With the Jacobian transformation in curvilinear coordinate system, Eq. (19) can be transformed into

$\frac{\partial T}{\partial t}=C_{1} \frac{\partial^{2} T}{\partial \xi^{2}}+C_{2} \frac{\partial^{2} T}{\partial \eta^{2}}+C_{3} \frac{\partial^{2} T}{\partial \xi \partial \eta}+C_{4} \frac{\partial T}{\partial \xi}+C_{5} \frac{\partial T}{\partial \eta}$

where

$$
\left\{\begin{array}{l}
C_{1}=\xi_{x}^{2}+\xi_{y}^{2} \\
C_{2}=\eta_{x}^{2}+\eta_{y}^{2} \\
C_{3}=2\left(\xi_{x} \eta_{x}+\xi_{y} \eta_{y}\right) \\
C_{4}=\xi_{x x}+\xi_{y y}+\xi_{y} / y \\
C_{5}=\eta_{x x}+\eta_{y y}+\eta_{y} / y
\end{array}\right.
$$

Heat conduction equations are typical parabolic equations, and plenty of schemes can be chosen for discrete such as FTCS, BTCS, Crank-Nicolson, improved Richardson scheme and so on. Most of the schemes are unconditionally stable, and the authors considered that the improved Richardson scheme is one of the most convenient and simplest schemes for numerical solutions of high efficiency.

Richardson constructed the first finite difference approach for the parabolic problem $u_{t}=\beta u_{x x}$ in 1910, which is named as Richardson 
scheme [5]. Richardson applied the central difference method for both temporal and spatial discretization, which can be written as

$\frac{u_{i}^{n+1}-u_{i}^{n-1}}{2 \Delta t}=\beta \frac{u_{i+1}^{n}-2 u_{i}^{n}+u_{i-1}^{n}}{\Delta x^{2}}$

The scheme has a 2 nd order of accuracy for both time and space. However, O'Brien et al. [6] demonstrated that, the Richardson scheme could not satisfy the von Neumann Stability Criteria in 1951. Du Fort and Frankel [7] successfully made improvement to the original Richardson scheme by replacing $u_{\mathrm{i}}^{\mathrm{n}}$ in the diffusion term with the average of $u_{\mathrm{i}}^{\mathrm{n}-1}$ and $u_{\mathrm{i}}^{\mathrm{n}+1}$. Furthermore, assuming $\sigma=\beta \Delta t / \Delta x^{2}$, the difference scheme can be rewritten as

$u_{\mathrm{i}}^{n+1}=\frac{2 \sigma}{1+2 \sigma}\left(u_{\mathrm{i}+1}^{n}+u_{\mathrm{i}-1}^{n}\right)+\frac{1-2 \sigma}{1+2 \sigma} u_{\mathrm{i}}^{n-1}$

This equation is named as improved Richardson scheme or Du Fort-Frankel scheme, with 2nd order accuracy of both time and space. Although there are three time levels appearing as $(n-1), n$ and $(n+1)$, it is still considered to be an explicit method due to simple construction shown in Eq. (21). Stability analysis demonstrates that this scheme is unconditionally stable, which is of great significance for the numerical treatment. The numerical algorithm is validated by solving the heat conduction in a $90^{\circ}$ sector annulus and comparing it with the theoretical solution in Eq. (17), and the boundary conditions

$$
\left\{\begin{array}{l}
\left.T\right|_{r=r_{1}}=0,\left.\frac{\partial T}{\partial r}\right|_{r=r_{2}}=\frac{\pi}{2}-\theta \\
\left.\frac{\partial T}{\partial r}\right|_{\theta=0}=\left.\frac{\partial T}{\partial r}\right|_{\theta=\pi / 2}=0
\end{array} .\right.
$$

Fig. 2(a) shows the temperature distribution on the outer boundary wall calculated by numerical simulation and theoretical formula, respectively. The numerical and theoretical solutions match with each other and indicate the credibility of the numerical algorithm. Fig. 2(b) displays the residual error between adjacent time steps and shows that a steady solution can be achieved by the numerical code with a few time steps.

With such a numerical algorithm, the heat conduction in the hollow semi-sphere can be simulated easily and compared with the series solution in Eq. (17). As mentioned at the end of the last chapter, the Legendre function could not be analytic at $\theta=\pi / 2$, and the solution may differ from reality at this location. Fig. 3 shows the comparison between the analytical and numerical solutions, for the range of $\theta$ from 0 two $0.4 \pi$. Here, the analytical and numerical solutions match with each other while the deflection is much more obvious between them at the range from $0.4 \pi$ to $\pi / 2$. It is up to the relaxation of the constraint by boundary condition in Eq. (11-b) due to the shortage of the Legendre function itself. However, heat transfer nearby the stagnation region $(\theta=0)$ is more important than that in other positions and calls for most of our attention. Hence, this analytic solution can still be used for further analysis and might satisfying the requirement of actual engineering applications. In the last comparison between analytical and numerical solutions, the heat transfer at outer wall is represented by $q_{\mathrm{w}}(\theta)=\pi / 2-\theta$, which is very easy for the integration in the series solution of Eq. (17) and much simpler than the hypersonic aerodynamic heating distribution. Therefore, the practicability of the analytical solution greatly depends on the difficulty of integration process. To simplify the integration, the hypersonic aerodynamic heating distribution governed by the local similarity solution should be applied with some special treatment.

\section{Approximation of hypersonic local similarity solution}

With the classic local similarity solution, the distribution of normalized heat transfer rates over the surface of spheres flying at supersonic speed [3] can be calculated as follows

$q_{\mathrm{w}}(\theta)=F_{1}\left(M_{\infty}, \theta\right)\left[\frac{1+0.096 \sqrt{\varepsilon}}{1.068}\right] \frac{1-g_{\mathrm{w}}}{1-g_{\mathrm{ws}}}$

where $\varepsilon$ stands for circumferential motion factor, and $g_{\mathrm{w}}$ and $g_{\mathrm{ws}}$ stands for wall enthalpy and stagnation wall enthalpy, respectively. In addition, the expression $F_{1}$ in Eq. (23) can be given as

$F_{1}\left(M_{\infty}, \theta\right)=2 \theta \sin \theta\left[\left(1-\frac{1}{\gamma_{\infty} M_{\infty}^{2}}\right) \cos ^{2} \theta+\frac{1}{\gamma_{\infty} M_{\infty}^{2}}\right]\left[D\left(M_{\infty}, \theta\right)\right]^{-\frac{1}{2}}$

while

$D\left(M_{\infty}, \theta\right)=\left(1-\frac{1}{\gamma_{\infty} M_{\infty}^{2}}\right)\left(\theta^{2}-\frac{\theta \sin 4 \theta}{2}+\frac{1-\cos 4 \theta}{8}\right)+\frac{4}{\gamma_{\infty} M_{\infty}^{2}}\left(\theta^{2}-\theta \sin 2 \theta+\frac{1-\cos 2 \theta}{2}\right)$

where $\gamma_{\infty}$ and $M_{\infty}$ represent specific heat ratio and Mach number of coming flow.

\section{a}

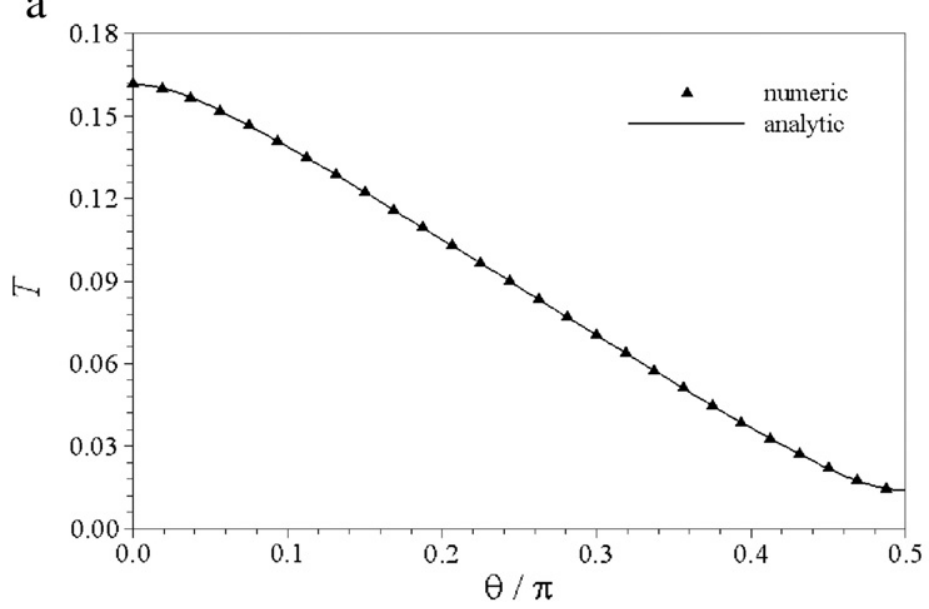

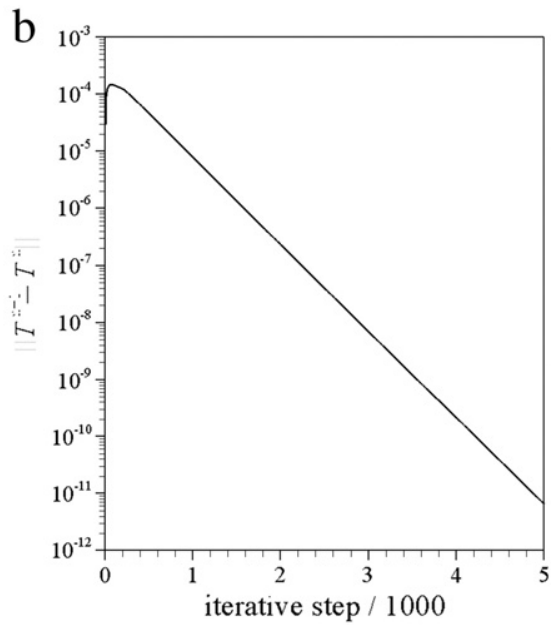

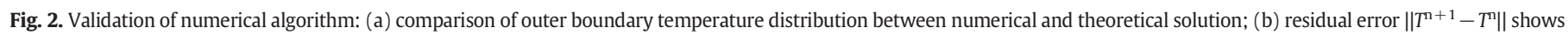
the convergence of numerical solution. 


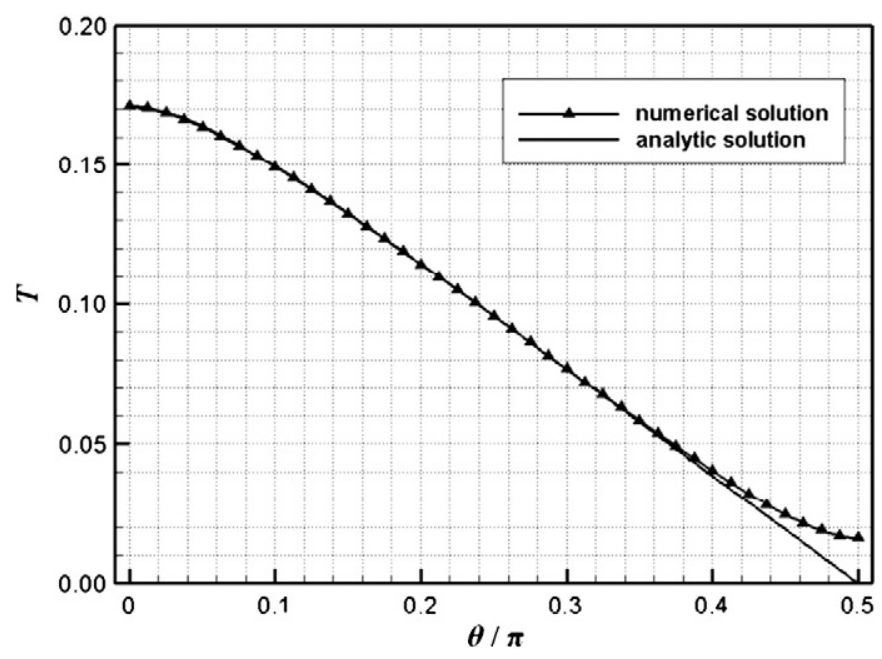

Fig. 3. Comparison of outer wall temperature distribution between analytical and numerical solutions with $r_{1}=0.4, r_{2}=0.5, T_{\mathrm{w}}(\theta)=0$ and $q_{\mathrm{w}}(\theta)=\pi / 2-\theta$.

At hypersonic speeds, the Mach number of the flow is usually higher than 5, which could be written as $M_{\infty} \geq 5$. Therefore, Eqs. (24) and (25) can be approximately simplified as

$F_{1}\left(M_{\infty}, \theta\right) \approx 2 \theta \sin \theta \cos ^{2} \theta\left(\theta^{2}-\frac{\theta \sin 4 \theta}{2}+\frac{1-\cos 4 \theta}{8}\right)^{-\frac{1}{2}}$

While circumferential motion factor and the enthalpy of wall and stagnation affect the amplitude of the absolute value, the function $F_{1}\left(M_{\infty}, \theta\right)$ plays a dominant role in governing the relative distribution of the aerodynamic heating on the outer wall. Hence, the distribution of normalized heat transfer rates for semi-spheres flying at hypersonic speed, where $q_{\mathrm{w}}$ equals 1 at the stagnation region, can be expressed as

$f(\theta)=2 \theta \sin \theta \cos ^{2} \theta\left(\theta^{2}-\frac{\theta \sin 4 \theta}{2}+\frac{1-\cos 4 \theta}{8}\right)^{-\frac{1}{2}}$

Eq. (27) needs to be substituted into Eq. (17) so that the analytical solution could be completed. However, if it was substituted into $\int_{0}^{\pi} q_{w}(\varphi) P_{n}(\cos \varphi) \sin \varphi d \varphi$ directly, the integration will be very complex and nearly impossible as the subscript $n$ keeps on increasing and approaches infinite. With observation of such an integration, we can easily get

$\int_{0}^{\pi} q_{\mathrm{w}}(\varphi) P_{\mathrm{n}}(\cos \varphi) \sin \varphi d \varphi=-\int_{0}^{\pi} q_{\mathrm{w}}(\varphi) P_{\mathrm{n}}(\cos \varphi) d \cos \varphi$

If $q_{\mathrm{w}}(\varphi)$ could be replaced by a polynomial of $\cos \varphi$, the integration process will be simple enough to handle. Since the coefficient calculated by the integration may be 0 due to the orthogonality of $P_{\mathrm{n}}(\cos \varphi)$, Eq. (17) can be simplified to a polynomial expression. The order of the polynomial expression will be equal to that of the polynomial expression of $q_{\mathrm{w}}(\varphi)$. Hence, a 4 th order polynomial of $\cos \theta$ defined as $g(\theta)$ is applied to approach the $f(\theta)$, and $g(\theta)$ can be written as

$g(\theta)=a_{1} \cos \theta+a_{2} \cos ^{2} \theta+a_{3} \cos ^{3} \theta+a_{4} \cos ^{4} \theta$

with the coefficients $a_{1}, a_{2}, a_{3}$ and $a_{4}$ calculated by the method of undetermined coefficients. Since $g(\theta)$ coincides with $f(\theta)$ permanently when $\theta=\pi / 2$, four other locations are chosen as reference points for the approximation as stated below

$$
\left\{\begin{array}{l}
g(0)=f(0)=1 \\
g(\pi / 4)=f(\pi / 4) \\
g(\pi / 3)=f(\pi / 3) \\
g(2 \pi / 5)=f(2 \pi / 5)
\end{array}\right.
$$

With the system of linear algebraic equations solved, the following equation is obtained

$g(\theta)=0.0207 \cos \theta+1.8855 \cos ^{2} \theta-1.2973 \cos ^{3} \theta+0.3911 \cos ^{4} \theta$

As an approximation by fitting with polynomial expressions, $g(\theta)$ approaches to $f(\theta)$ and matches with it well, as shown in Fig. 4. Therefore, the distribution of hypersonic aerodynamic heating governed by the local similarity solution can be totally replaced by such an approximate solution, which will lower the difficulty in completing the integration in Eq. (17).

Since both of the series solutions in Eq. (17) and $g(\theta)$ are polynomial expressions of $\cos \theta$ and $g(\theta)$ is just of the 4th order, $g(\theta)$ can also be expanded in the form of the Legendre polynomials no more than the 4th order, which will lead to further simplification for the series solution and make the process more convenient. The expansion can be shown as follows

$g(\theta)=\sum_{\mathrm{n}=0}^{4} A_{\mathrm{n}} P_{\mathrm{n}}(\cos \theta)$

with

$$
\left\{\begin{array}{l}
P_{0}(\cos \theta)=1 \\
P_{1}(\cos \theta)=\cos \theta \\
P_{2}(\cos \theta)=\frac{1}{2}\left(3 \cos ^{2} \theta-1\right) \\
P_{3}(\cos \theta)=\frac{1}{2}\left(5 \cos ^{3} \theta-3 \cos \theta\right) \\
P_{4}(\cos \theta)=\frac{1}{8}\left(35 \cos ^{4} \theta-30 \cos ^{2} \theta+3\right)
\end{array}\right.
$$

The coefficients $A_{\mathrm{n}}$ in Eq. (32) can be determined by comparing with the coefficients in Eq. (31), which results into

$\left\{\begin{array}{l}A_{0}=0.70762 \\ A_{1}=-0.75768 \\ A_{2}=1.48049 \\ A_{3}=-0.51892 \\ A_{4}=0.089394\end{array}\right.$

Based on this, the analytical series solution in Eq. (17) is just of the 4th order, and replaces the determination of coefficients by the integration $\int_{0}^{\pi} q_{\mathrm{w}}(\varphi) P_{\mathrm{n}}(\cos \varphi) \sin \varphi d \varphi$ with some constants shown in Eq. (33). The analytical solution can be approached by the assumptions and

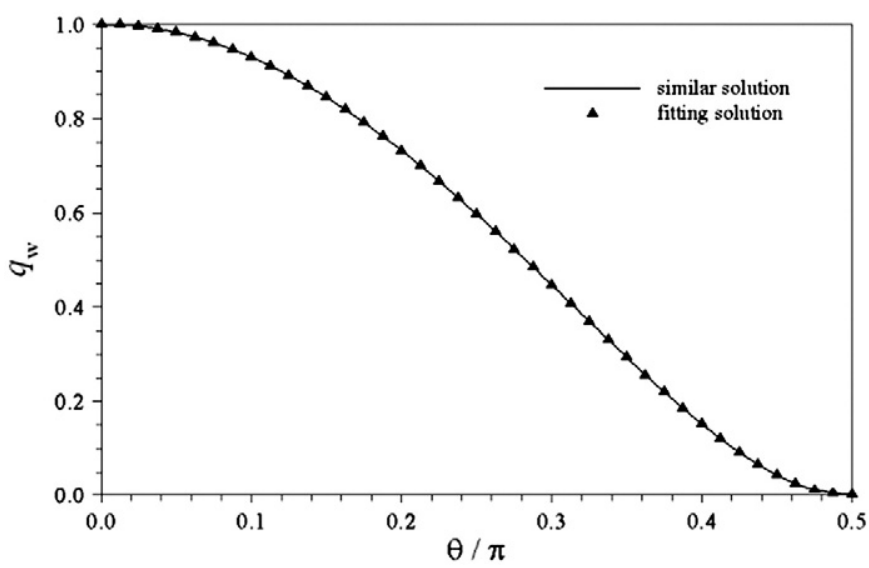

Fig. 4. Comparison between similar solution $f(\theta)$ and its fitting approximate solution $g(\theta)$. 
modifications mentioned above, and the approximate solution for the temperature field can be shown as

$T(r, \theta)=\sum_{n=0}^{4} A_{\mathrm{n}} \frac{2 n+1}{2}\left[\left(\frac{r}{r_{1}}\right)^{n}-\left(\frac{r_{1}}{r}\right)^{n+1}\right] P_{\mathrm{n}}(\cos \theta) \frac{r_{2} \alpha^{-n}}{n+(n+1) \alpha^{-(2 n+1)}}$

\section{Discussions}

The approximate analytic solution of heat conduction in hollow semi-sphere flying at hypersonic speed with inner active cooling has been set up with 4 th order Legendre polynomial approaches the local similarity solution of hypersonic aerodynamic heating. In order to validate the approximate solution and perform further analysis of the heat conduction, the distribution of temperature on the outer boundary and heat transfer rate on the inner boundary, and comparison on these between analytical and numerical solutions will be shown as below.

\subsection{Temperature on outer boundary}

For hypersonic flight, the structure should afford the serious aerodynamic heating loads for the requirement of safety. The structural temperature of the structure should be lower than a critical value, which may result in damages to the performance of material, structure, and surface coating etc. Therefore, the temperature distribution on the outer boundary needs to be paid more attention. The temperature distribution on the outer wall can be given as

$\left.T\right|_{r=r_{2}}=\sum_{n=0}^{4} A_{n} \frac{2 n+1}{2} \frac{\alpha^{2 n+1}-1}{n\left(\alpha^{2 n+1}+1\right)+1} r_{2} P_{n}(\cos \theta)$

Eq. (35) indicates that the outer wall temperature is proportional to the radius $r_{2}$. Hence, the relationship between temperature and dimension of the structure is absolutely linear. However, the aerodynamic heating is not independent on dimension of the structure, which could be generalized as that the stagnation heat transfer rate is proportional to $r_{2}^{-1 / 2}$. Therefore, the temperature on the outer wall boundary and effect of dimension need further discussions, which the authors would not do in this paper. The comparison between analytical and numerical results of the outer wall temperature can be shown in Fig. 5, with the $r_{1}=0.4$, $r_{2}=0.5$, and $\alpha=1.25$. The analytical solution matches well with the numerical simulation result and the deflection nearby $\theta=\pi / 2$ is not so significant, which is totally different from that in Fig. 3. Thus, the idea and method have been done in this paper to get another demonstration.

Usually, the temperature at the stagnation region is considered to be an important parameter for the design of hypersonic vehicles. By Eq. (35), we can set $\theta$ to be zero standing for the stagnation. With $P_{\mathrm{n}}(1)=1$, the wall temperature at the outer stagnation point can be derived as

$\left.T\right|_{r=r_{2}, \theta=0}=\sum_{n=0}^{4} A_{n} \frac{2 n+1}{2} \frac{\alpha^{2 n+1}-1}{n\left(\alpha^{2 n+1}+1\right)+1} r_{2}$

Wall stagnation temperature of stagnation can be considered as a special case of temperature distribution on the outer boundary. We set the dimension of the inner radius $r_{1}=1$, and show the tendency of temperature change with $r_{2}=\alpha r_{1}$. The analytical and numerical results match with each other, which should be a demonstration of the dimension effect, as shown in Fig. 6.

\subsection{Heat transfer rate on inner boundary}

As a part of thermal protection systems, active cooling systems assumed before call us for more investigations on the heat exchange on

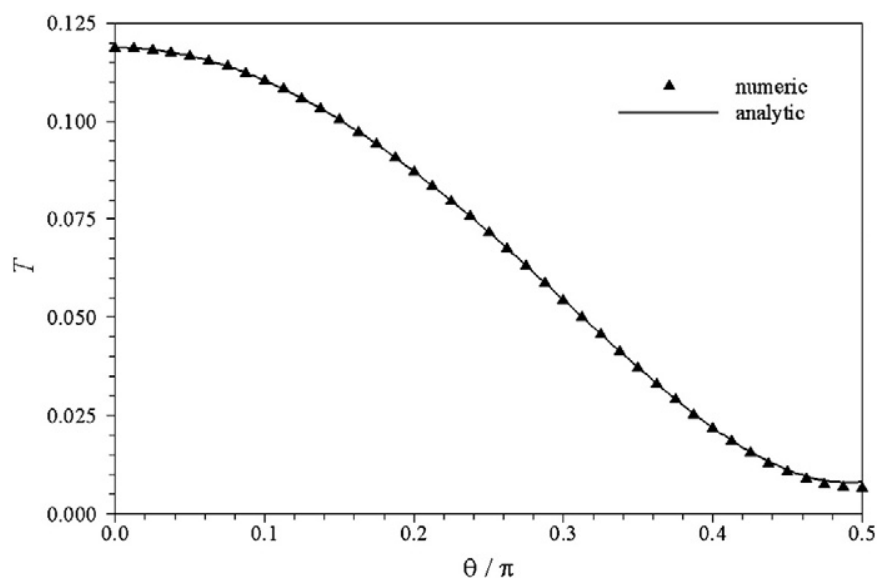

Fig. 5. The temperature distribution on the outer boundary calculated by both analytical and numerical solution with $r_{1}=0.4, r_{2}=0.5, T_{\mathrm{w}}(\theta)=0$ and $q_{\mathrm{w}}(\theta)=\mathrm{g}(\theta)$.

the inner boundary. Based on the discussions in the foregoing chapters, the heat transfer rate on the inner boundary, defined as the partial differential of temperature over radius, can be derived as follows

$\left.\frac{\partial T}{\partial r}\right|_{r=r_{1}}=\sum_{n=1}^{4} A_{\mathrm{n}} \frac{(2 n+1)^{2}}{2} \frac{\alpha^{n+2}}{n\left(\alpha^{2 n+1}+1\right)+1} P_{\mathrm{n}}(\cos \theta)$

With further observation of Eq. (37), it is easy to find out that the heat transfer rate is dependent on the relative dimension $\alpha=r_{2} / r_{1}$ rather than the absolute dimension of the hollow semi-sphere. It is also necessary to figure out the heat transfer rate at the inner stagnation region, which can be defined as

$\left.\frac{\partial T}{\partial r}\right|_{r=r_{1}, \theta=0}=\sum_{n=1}^{4} A_{n} \frac{(2 n+1)^{2}}{2} \frac{\alpha^{n+2}}{n\left(\alpha^{2 n+1}+1\right)+1}$

The comparison of heat transfer rate at inner stagnation region between analytical and numerical results is shown in Fig. 7, where both of them match well with each other. With an increase of $\alpha$ from 1.0 to $1.5, q_{\mathrm{ws}}$ on the inner boundary increases to about 1.75 . However, for active cooling system, the structure should not be too thick, which means $\alpha$ might not be higher than 1.2 and the corresponding $q_{\text {ws }}$ on the inner boundary is no more than 1.4. Therefore, it can be used to analyze cases like this.

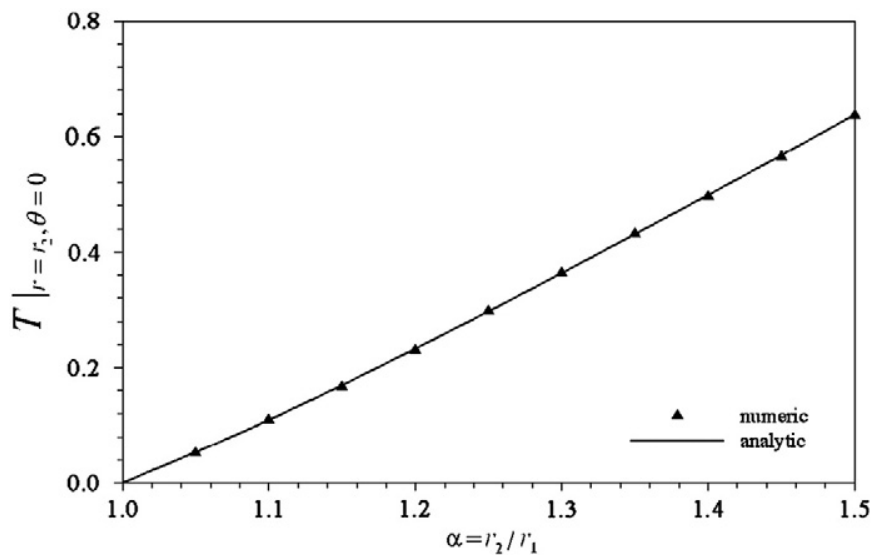

Fig. 6. Wall temperature at outer stagnation changes with increase of $\alpha$ when $r_{1}=1$, $T_{\mathrm{w}}(\theta)=0$ and $q_{\mathrm{w}}(\theta)=\mathrm{g}(\theta)$. 


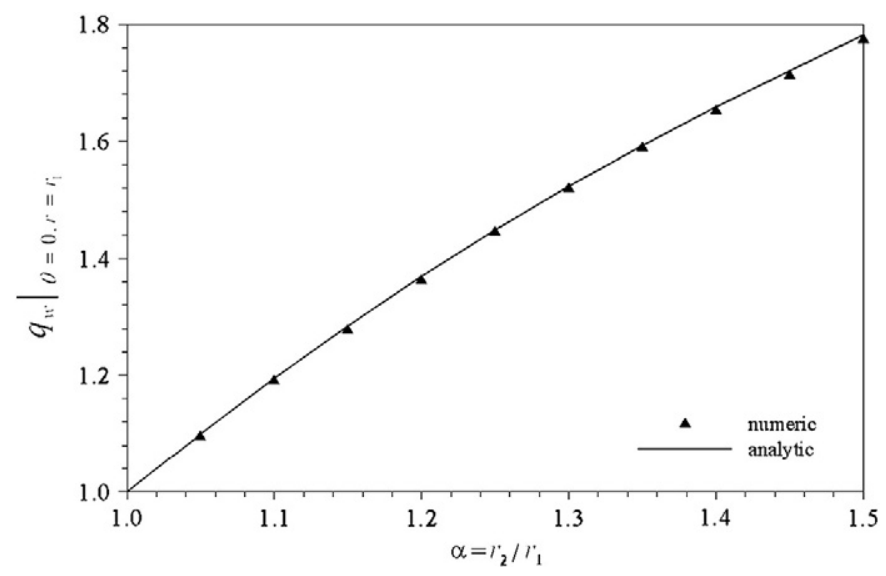

Fig. 7. Heat transfer rate at inner stagnation changes with increase of $\alpha$ when $r_{1}=1$, $T_{\mathrm{w}}(\theta)=0, q_{\mathrm{w}}(\theta)=\mathrm{g}(\theta)$.

\section{Conclusions}

This paper has set up an approximate analytic solution of heat conduction in hollow semi-spheres flying at hypersonic speed. The heat conduction equation was solved by separation of variables and application of the Legendre function, and the series solution is of course in the form of the Legendre function. However, the integration in the analytical solution can be hardly performed due to the complexity of the local similarity solution of hypersonic aerodynamic heating. With the observation of the analytical solution, a 4th order polynomial of cosine function is applied to approach the local similarity solution, which could be equivalently transformed into a 4th order series of the Legendre function. Hereby, the approximation and transformation make the integration in the analytical solution possible and easy, and lead to an approximate analytical solution. With further validation by numerical simulations, the approximate analytical solution was observed to match well with the numerical results and accord with physical laws and phenomena. Furthermore, this paper primarily discussed the wall temperature and heat transfer rate on outer and inner boundaries respectively, which could be considered as applications of the approximate analytical solution. In actual active systems, the boundary conditions are very difficult to set up and modeled, especially the inner boundary conditions. This paper just made some direct assumption, a general form of temperature distribution on the inner wall, to make the problem possible to be solved. However, the exact expression of the temperature distribution needs more discussion and validation case by case. They need more investigations for hypersonic applications in the coming future.

\section{Acknowledgements}

Thanks to the support of the National Natural Science Foundation of China (No. 90916028).

\section{References}

[1] J.J. Bertin, R.M. Cummings, Critical hypersonic aerodynamic phenomenon, Annual Review of Fluid Mechanics 38 (2006) 129-157.

[2] F.Q. Zhong, X.J. Fan, G. Yu, et al., Heat transfer of aviation kerosene at supercritical conditions, Journal of Thermophysics and Heat Transfer 23 (3) (2009) 543.

[3] N.H. Kemp, P.H. Rose, R.W. Detra, Laminar heat transfer around blunt bodies in dissociated air, Journal of Aerospace Sciences 26 (7) (1959).

[4] H. Bateman, Higher Transcendental Functions, McGraw-Hill Book Company, INC, 1953.

[5] L.F. Richardson, The approximate arithmetical solution by finite differences of physical problems involving differential equations, with an application to the stress in a masonry dam, Philosophical Transactions of the Royal Society of London, Series A 210 (1910) 307-357, (and Proc. Roy. Soc. London, Ser. A, 1910, 83: 335-356).

[6] G.G. O'Brien, M.A. Hyman, S. Kaplan, A study of the numerical solution of partial differential equations, Journal of Mathematical Physics 29 (1951) 223-251.

[7] E.C. Du Fort, S.P. Frankel, Stability conditions in the numerical treatment of parabolic differential equations, Mathematical Tables and Other Aids to Computation 7 (1953) $135-152$. 